\title{
Beam Parameter Product Optimization for High Power SWIR Laser Diode Stack Fiber Coupling
}

\author{
Yves Lutz \\ Advanced Visionic and Processing, French-German Research Institute, Saint-Louis, France \\ Email: Yves.lutz@isl.eu
}

How to cite this paper: Lutz, Y. (2022) Beam Parameter Product Optimization for High Power SWIR Laser Diode Stack Fiber Coupling. Optics and Photonics Journal, 12, 19-29.

https://doi.org/10.4236/opj.2022.122002

Received: January 12, 2022

Accepted: February 25, 2022

Published: February 28, 2022

Copyright (c) 2022 by author(s) and Scientific Research Publishing Inc. This work is licensed under the Creative Commons Attribution International License (CC BY 4.0).

http://creativecommons.org/licenses/by/4.0/ (c) (i) Open Access

\begin{abstract}
An optimized setup for fiber optic injection of a kilowatt peak power laser diode stack emitting in the SWIR spectral range is proposed. Starting from a fast axis collimated (FAC) and slow axis collimated (SAC) 15 bars, 19 emitters off the shelf laser diode stack, the beam is transformed using spatial beam combining and polarization coupling. Both techniques integrated in a compact design enable to couple the kilowatt level beam into a standard $600 \mu \mathrm{m}$ core, 0.22 numerical aperture (NA) multimode optical fiber. An application in the field of long range SWIR laser illuminator for gated viewing is presented. A comparison between two illuminators is realized both based on the same laser diode stack but one using beam parameter product (BPP) reduction and one without. It could be demonstrated that BPP reduction is the best way for efficient, narrow divergence and compact semi-conductor based laser illuminators design and realization. The global laser illuminator efficiency could be improved by $75 \%$ for the narrowest divergences thanks to this approach.
\end{abstract}

\section{Keywords}

Semiconductor Laser, Beam Shaping, Beam Parameter Product, Spatial Multiplexing, Polarization Coupling, Laser Illuminator

\section{Introduction}

Pulsed high repetition rate, medium average power illuminators are needed for eye safe and long range active imaging applications [1] [2] [3]. The semi-conductor laser is the most adapted and most efficient laser source in this field [4] but, unfortunately, the direct use is unsuitable due to its poor beam quality especially in the case of high power stacked arrays [5] [6]. One key way to overcome this difficulty is to couple the beam into a multimode optical fiber which 
acts as a homogenizer and allows a simplified beam treatment for divergence control or focusing. The challenge is to optimize the coupling efficiency as well as the brightness. The dimensions of the lighting optics are directly related to the brightness of the beam, which is why special priority must be given to this point.

The needed power for long range illuminators leads to select high power stacked laser diode bars. Nowadays, 70 W peak power bars @1550 nm are currently available [7]. To reach the kilowatt range, a stack with 15 bars composed of 19 emitters each is selected. To allow conduction cooling, the pitch between each bar is equal to $1.6 \mathrm{~mm}$ resulting in a total emitting area of $24 \mathrm{~mm} \times 10 \mathrm{~mm}$. The divergences of both fast and slow axis are reduced to $15 \mathrm{mrad}$ and $35 \mathrm{mrad}$ respectively by using micro lens arrays provided by the manufacturer. In such a configuration, the BPP of both axes is close together which allows a direct fiber coupling like our first developed system presented in [8]. Unfortunately, without brightness optimization, the coupling efficiency as well as the divergence handling of the laser illuminator remains limited, especially for narrow fields. Indeed, improving brightness can considerably increase fiber coupling and relax the requirements of the collimating optics used to control the divergence of a laser illuminator. This complete optimization process is described and experimented in this paper with the support of simulation.

\section{Beam Shaping Operations}

\subsection{Spatial Beam Multiplexing}

\subsubsection{Simulation}

The first operation applied to the beam directly after the micro lens array consists of virtually filling the empty spaces between each bar as proposed by Ghasemi [9] and illustrated on Figure 1. Simulations show that this operation is possible with our 15 bars stack without occulting any part of the individual beams.

\subsubsection{Experimentation}

A first experimental setup is built with two mirrors as illustrated on Figure 2(a). The first mirror M1 consists of a first face with AR coating placed in front of the laser diode stack with an angle of $45^{\circ}$ and a second face equipped with 7 high reflection stripes to reflect the beam coming from M2. The second mirror M2 with high reflection coating at $45^{\circ}$ redirects the transmitted beams from the 7 upper bars to the stripe mirror M1. Power measurements have been conducted on the different points and the transmission of the different steps are deduced and summarized on Table 1. The experimental conditions are the follow; laser power at maximum with drive current of $180 \mathrm{~A}$ ng to $1000 \mathrm{~W}$ peak power with pulse duration of $5 \mu$ s at a repetition rate of $1 \mathrm{kHz}$.

As it can see on Table 1(a), the transmission of the beam by the first mirror is limited to $89 \%$. It should be mentioned that unlike the first diopter of the M1 mirror, the second face which contains the stripe coatings is not AR coated 




Figure 1. Spatial beam multiplexing by using step mirrors.

mainly for simplification reasons. With such an additional operation the transmission could be improved in this configuration. Another origin for losses can be attributed to the polarization losses due to the sagittal linear polarization of the stack. In our design presented on Figure 2, the incident angle of the polarized beam gets closer to the Brewster angle of our mirror $\left(57^{\circ}\right)$ but unfortunately with 


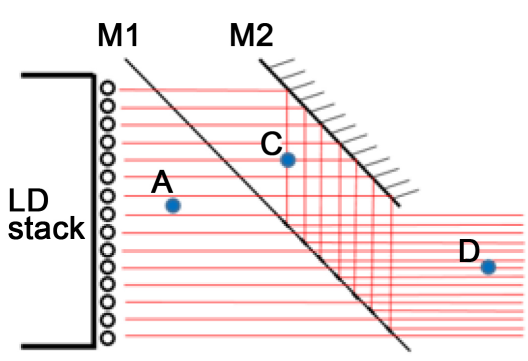

(a)

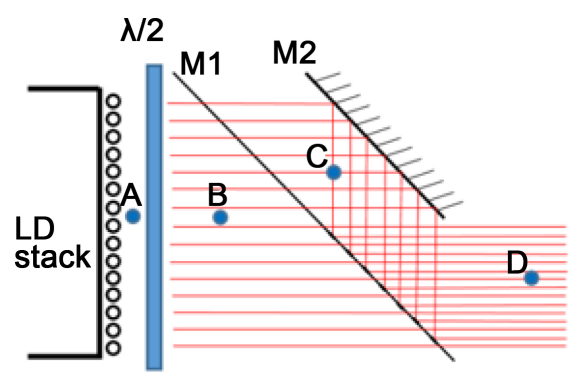

(b)

Figure 2. Spatial beam multiplexing setup with (b) and without (a) polarization rotating, measurement positions are filled in blue.

Table 1. Transmission efficiencies with (b) and without (a) lambda half wave plate.

\begin{tabular}{|c|c|c|c|c|c|c|c|c|}
\hline & \multicolumn{3}{|c|}{ Case (a) } & & \multicolumn{4}{|c|}{ Case (b) } \\
\hline & $\mathrm{C} / \mathrm{A}$ & $\mathrm{D} / \mathrm{C}$ & $\mathrm{D} / \mathrm{A}$ & & $\mathrm{B} / \mathrm{A}$ & $\mathrm{C} / \mathrm{B}$ & $\mathrm{D} / \mathrm{C}$ & $\mathrm{D} / \mathrm{A}$ \\
\hline $\mathrm{T}[\%]$ & 89 & 97 & 86.3 & $\mathrm{~T}[\%]$ & 98 & 98 & 97 & 93.1 \\
\hline
\end{tabular}

the polarization configuration perpendicular to incident plane corresponding to the highest losses. On the other hand, only $3 \%$ of losses are measured by the beam redirecting M2 mirror, this result definitively validate the HR stripe design.

In a second step a zero order lambda half wave plate @1550 nm (see Figure $2(b))$ is introduced in order to reduce the losses. The result of this operation can be shown on Table 1(b). The global transmission of this complete first beam shaping operation could be improved from $86.3 \%$ to $93.1 \%$. The laser diode stack polarization degree is very high allowing an efficiency of $98 \%$ by polarization rotating. After that, the Brewster losses could be dramatically reduced without any degradation by beam redirecting with M2. At this point, the BPP in the fast axis direction could be reduced by a factor of two. Figure 3 illustrates the beam profile directly at the stack output and after the first BPP reduction stage. For all beam profile measurements, the drive current of the stack is set to a value just above the threshold current to avoid damaging the camera. The next step is to work on the slow axis BPP reduction before fiber injection.

\subsection{BPP Reduction along the Slow Axis}

In order to reduce the BPP along the slow axis, the technique of polarization coupling is chosen [10]. This technique is often used to couple two stacks together however, in our case, the idea is to overlap two sub-beams after separation as illustrated on Figure 4.

After spatial beam multiplexing, the resulting beam is cut into two sub-beams of equal power with a sharp edge high reflection mirror M3. The first sub beam is directed to the coupling polarization prism without polarization change whereas the second one undergoes a new polarization rotation of $90^{\circ}$ with a second 

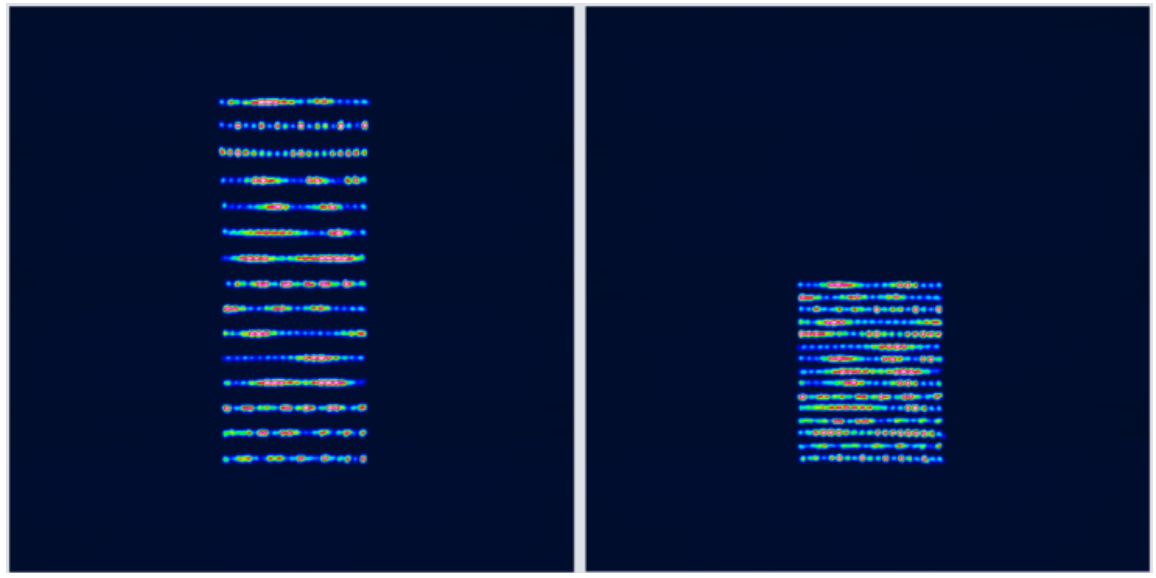

Figure 3. Measured beam profile at the output of the stack (position A) left and at the output of the spatial multiplexing stage (position $\mathrm{D}$ ) right.

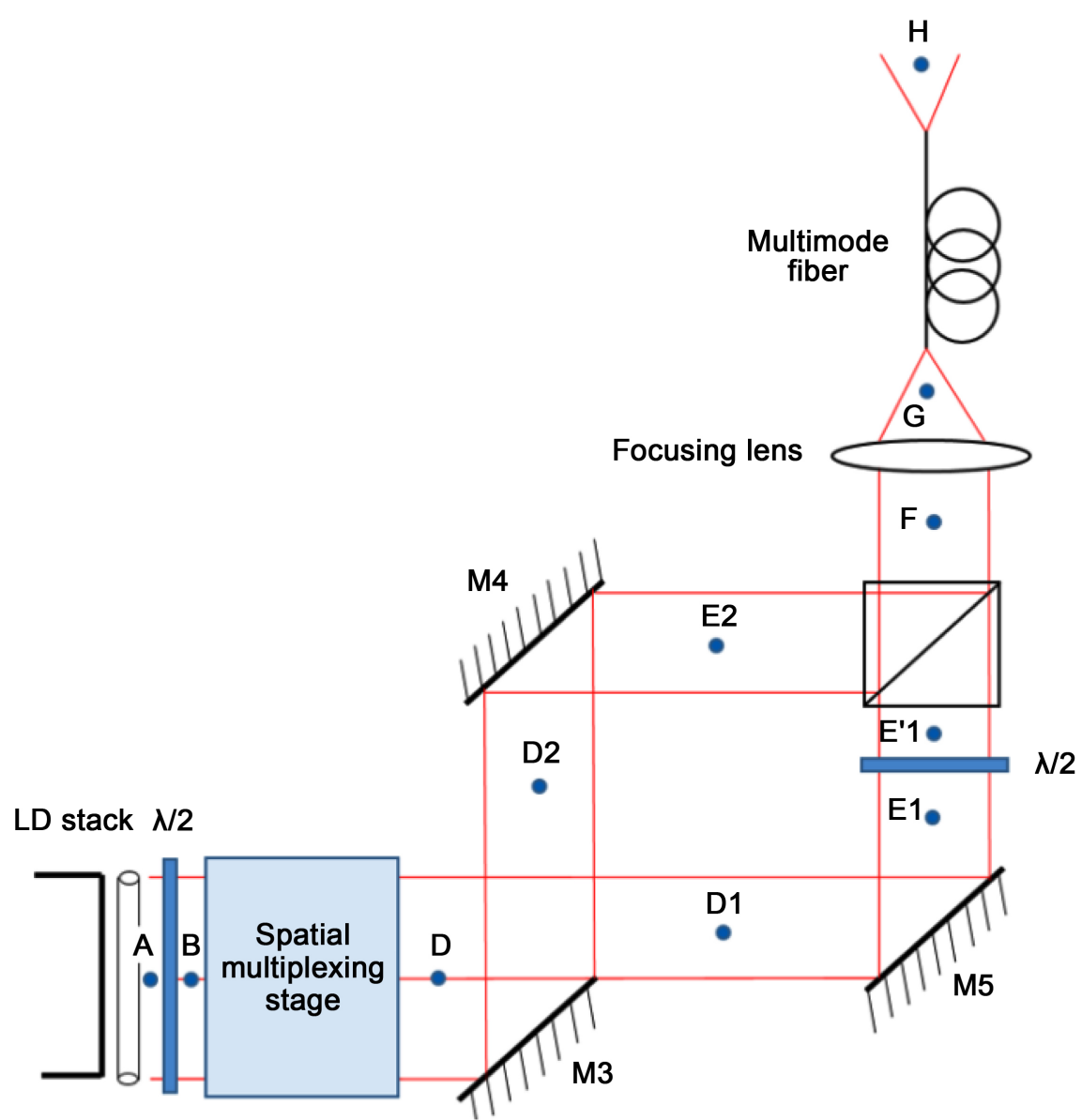

Figure 4. Setup for BPP reduction and fiber coupling with the different measurement positions.

lambda half wave plate placed after mirror M5. For an efficient superposition, both optical ways are carefully equalized.

Figure 5 shows the profiles of the sub-beams recorded at position D1 and D2 and the superposition on F. Considering that the bars are composed of 19 


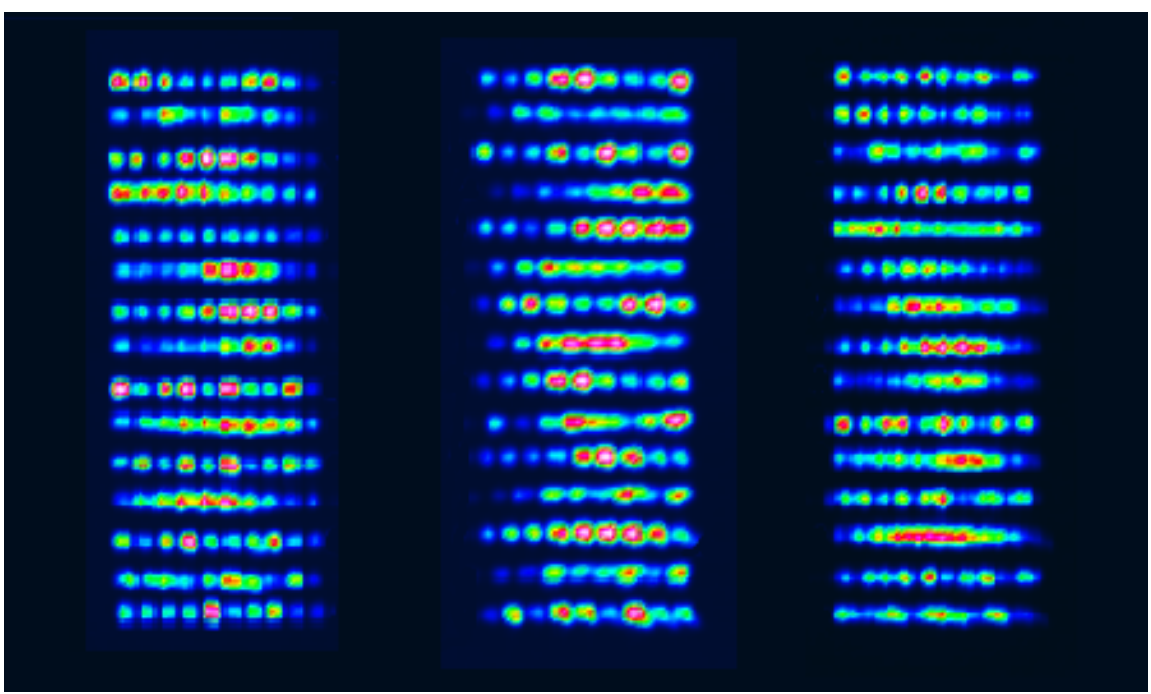

Figure 5. Measured beam profiles on positions D1 (left), D2 (center) and F (right), respectively.

emitters, the superposition of the two sub-beams results to equivalent bars with 10 emitters with cross polarization state.

In terms of efficiency, Table 2 represents the results of measurements of this complete polarization coupling stage. As it can be seen, the efficiency of this operation is very high, this can be explained by the high quality coating used and also on the high polarization state of the beam after spatial multiplexing. After this stage, the BPP of both axes could be reduced to $45 \times 43.5 \mathrm{~mm}$. mrad which represents the initial goal before fiber injection.

\subsection{Fiber Injection}

Focalization of the beam into a multimode fiber needs to respect both, the core diameter as well as the acceptance angle given by the numerical aperture. According to Yulong $\mathrm{Wu}$ [11], the following rules must be respected for efficient fiber coupling:

$$
\begin{aligned}
& \mathrm{BPP}_{\text {fiber }} \geq \sqrt{2} \times \mathrm{BPP}_{\mathrm{FA}} \\
& \mathrm{BPP}_{\text {fiber }} \geq \sqrt{2} \times \mathrm{BPP}_{\mathrm{SA}}
\end{aligned}
$$

With

$$
\mathrm{BPP}_{\text {fiber }}=\frac{\mathrm{D}_{\text {fiber }} \times \mathrm{NA}}{2}
$$

Table 3 gives the different multimode fibers used for beam injection and their corresponding BPP's. Only the $550 \mu \mathrm{m}$ core fiber with NA $=0.22$ does not match to the perfect injection conditions.

Several injection lenses have also been simulated and tested with focal lengths from 20 to $35 \mathrm{~mm}$. In order to minimize focusing aberrations, aspheric lenses as well as doublets and triplets where used. The best result is obtained with an AR coated aspheric lens with a focal length of $26 \mathrm{~mm}$ and a NA $=0.52$. The corresponding focal point is measured and presented on Figure 6 . In this case the 
Table 2. Transmission efficiencies of the spatial polarization BPP reduction stage.

\begin{tabular}{ccccc}
\hline & $(\mathrm{D} 1+\mathrm{D} 2) / \mathrm{D}$ & $(\mathrm{E} 1+\mathrm{E} 2) /(\mathrm{D} 1+\mathrm{D} 2)$ & $\mathrm{F} /(\mathrm{E} 1+\mathrm{E} 2)$ & $\mathrm{F} / \mathrm{D}$ \\
\hline $\mathrm{T}[\%]$ & 99 & 98 & 98 & 95 \\
\hline
\end{tabular}

Table 3. Core diameter, NA and corresponding BPP of the tested multimode fibers.

\begin{tabular}{ccccc}
\hline Core & $550 \mu \mathrm{m}$, & $600 \mu \mathrm{m}$, & $600 \mu \mathrm{m}$, & $1000 \mu \mathrm{m}$, \\
diameter, NA & $\mathrm{NA}=0.22$ & $\mathrm{NA}=0.22$ & $\mathrm{NA}=0.39$ & $\mathrm{NA}=0.22$ \\
\hline $\mathrm{BPP}_{\text {fiber }}$ & $61 \mathrm{~mm} \cdot \mathrm{mrad}$ & $66 \mathrm{~mm} \cdot \mathrm{mrad}$ & $120 \mathrm{~mm} \cdot \mathrm{mrad}$ & $101 \mathrm{~mm} \cdot \mathrm{mrad}$ \\
\hline
\end{tabular}

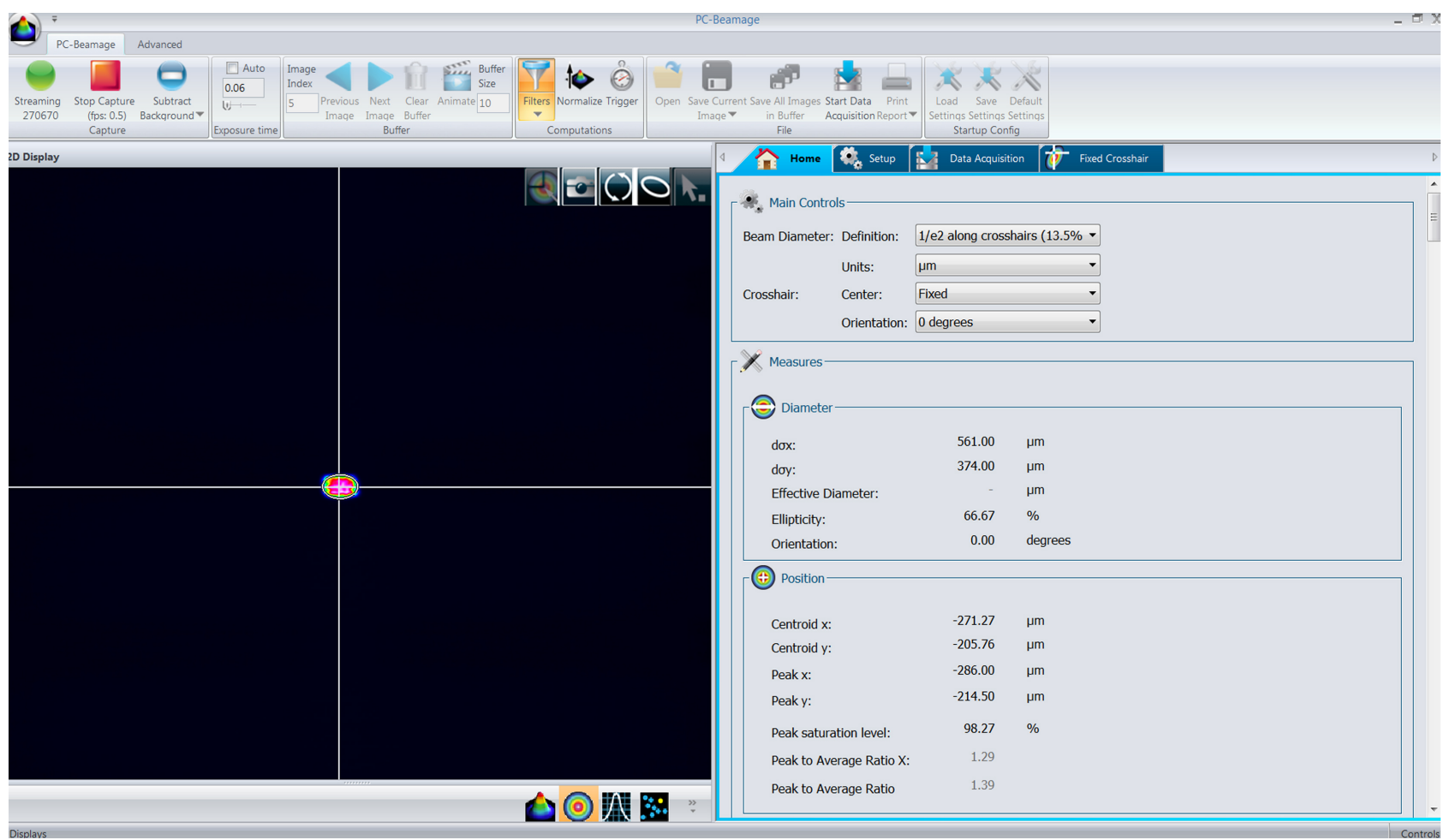

Figure 6. Beam profile on the focal point at maximum power.

power of the laser diode is set at the maximum close to $1 \mathrm{~kW}$. It can be seen that the beam remains elliptical (ellipticity $=66.67 \%$ ), nevertheless, the measured dimensions show that $86.5 \%$ of the global energy is contained in an ellipse of $561 \times$ $374 \mu \mathrm{m}$.

Figure 7 gives the measured power at the fiber output as a function of the diode drive current. As expected, the best result is obtained with the large core $1000 \mu \mathrm{m}$ fiber. The three other fibers show very close results with still the lowest value corresponding to the $550 \mu \mathrm{m}$ core fiber. Interesting is to mention that there is no real difference between NA of 0.22 and 0.39 comforting the fact that the injection angle is right. A slight slackening of the curves can also be observed for the highest powers which can be attributed to the spatial broadening with increased pump current. As mentioned, the goal is to keep the BPP as low as possible at the fiber output, it is why the fiber with $600 \mu \mathrm{m}$ core diameter and 
Fiber injection

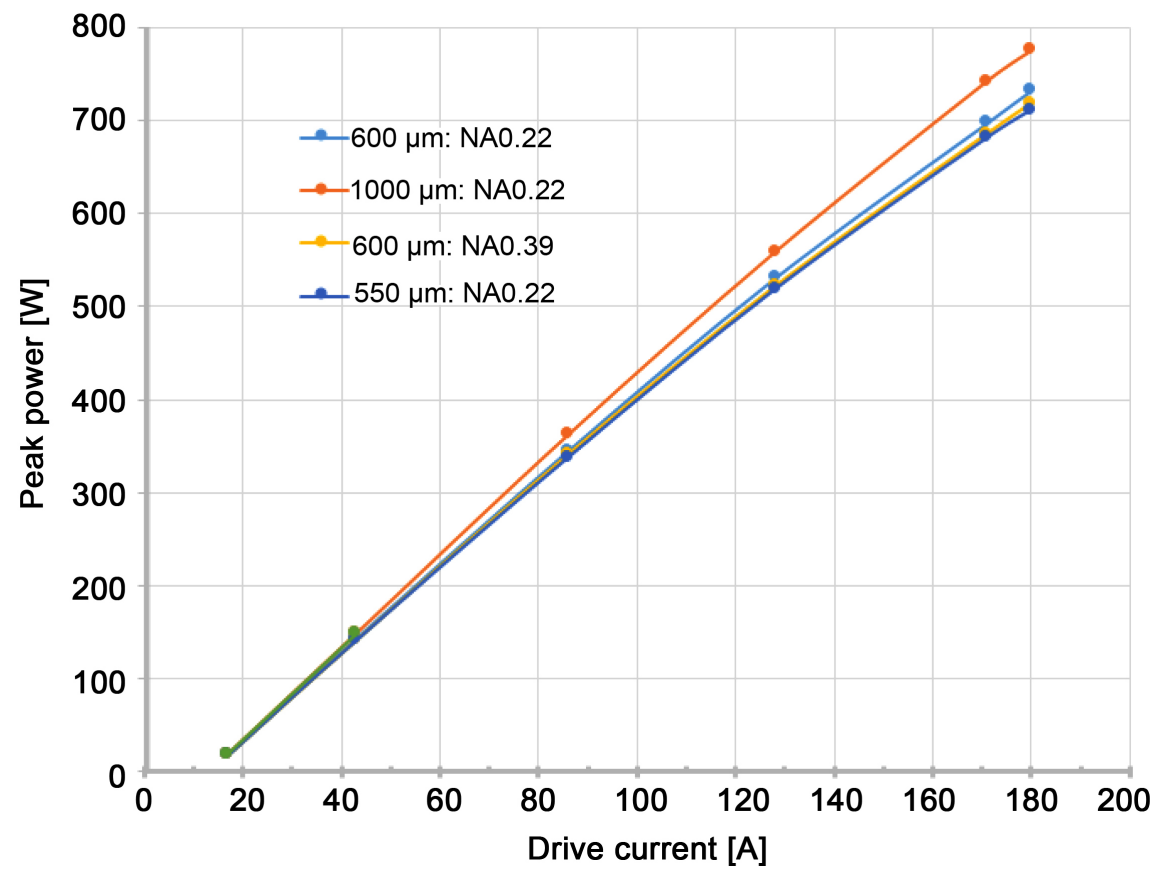

Figure 7. Optical fiber output power (position $\mathrm{H}$ ) versus drive current for the tested multimode fibers.

NA 0.22 represents the best compromise. With this fiber, the available output power reach $733 \mathrm{~W}$ with a coupling efficiency of $83 \%$. Optical fibers equipped with AR coatings on both side are suited for further coupling efficiency enhancement.

\section{Applications for Long Range Laser Illuminators}

For long range gated viewing applications, a first laser diode illuminator was built [8] based on the same stack described in this paper. Figure 8 gives the setup used for this first design.

Considering that the BPP's for the stack are very close together, only two cylindrical lenses are used to inject the beam into the multimode fiber. Here, the fiber has a core diameter of $1500 \mu \mathrm{m}$ with $\mathrm{NA}=0.22$. Without optical coatings the reached coupling efficiency was $89 \%$. In a second step a rectangular light pipe with a cross section of $1.5 \times 2 \mathrm{~mm}$ is used for beam forming in order to reach a perfect overlap between the illumination beam and the recorded image. Lens L3 projects the beam on the scene, the focal length gives the global divergence. To have a tunable divergence, a commercially available SWIR zoom lens with following characteristics is used:

$$
\begin{gathered}
25 \mathrm{~mm} \leq \mathrm{f} \leq 300 \mathrm{~mm} \\
2.8 \leq \mathrm{f} \# \leq 3.9
\end{gathered}
$$

Transmission at $1550 \mathrm{~nm}=65 \%$.

With this setup, the illuminator divergence has a tuning range of $0.29^{\circ} \times 0.38^{\circ}$ 
to $3.4^{\circ} \times 4.5^{\circ}$. The power on the target is measured as a function of the beam divergence and presented on Figure 9. An additional focusing lens is placed after the zoom lens for measurement purposes. Power on the target is measured at the focal point of this lens.

The curve starts with a power close to $550 \mathrm{~W}$ and drops with the highest focal length to value of $270 \mathrm{~W}$. This is due as much to the poor overall transmission of the zoom lens as well as to the limited optical aperture of the lens for the highest focal length values. The output pupil diameter is limited to $\phi=77 \mathrm{~mm}$ whereas with the used optical fiber and light pipe, the corresponding output pupil which can be deduced from a ray tracing, should have been $\phi \geq 135 \mathrm{~mm}$.

$$
\phi \geq 2 f \theta
$$

with $\theta=\sin ^{-1} \mathrm{NA}, f$ the focal length of the zoom lens and NA the numerical aperture of the fiber. With such a value, the global size of the illuminator should be largely increased leading in a significant degradation of the global compactness. On the other hand, no SWIR optical zoom lens could be found on the market with such an optical aperture and custom realization is a wrong way because of costs increasing. Thus, the best way is to reduce the brightness of the beam before hitting the zoom optic.

Figure 10 presents the ray tracing of the new illuminator design based on the

L1

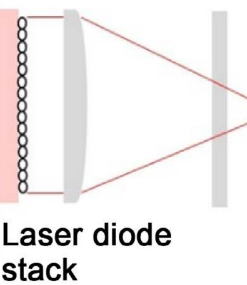

L2

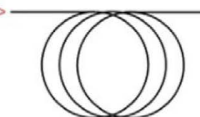

multimode fiber
L3

\section{Illuminator}

divergence $=\mathrm{FOV}$

rectangula light pipe

\section{cold plate}

Figure 8. First developed laser diode stack based long range illuminator.

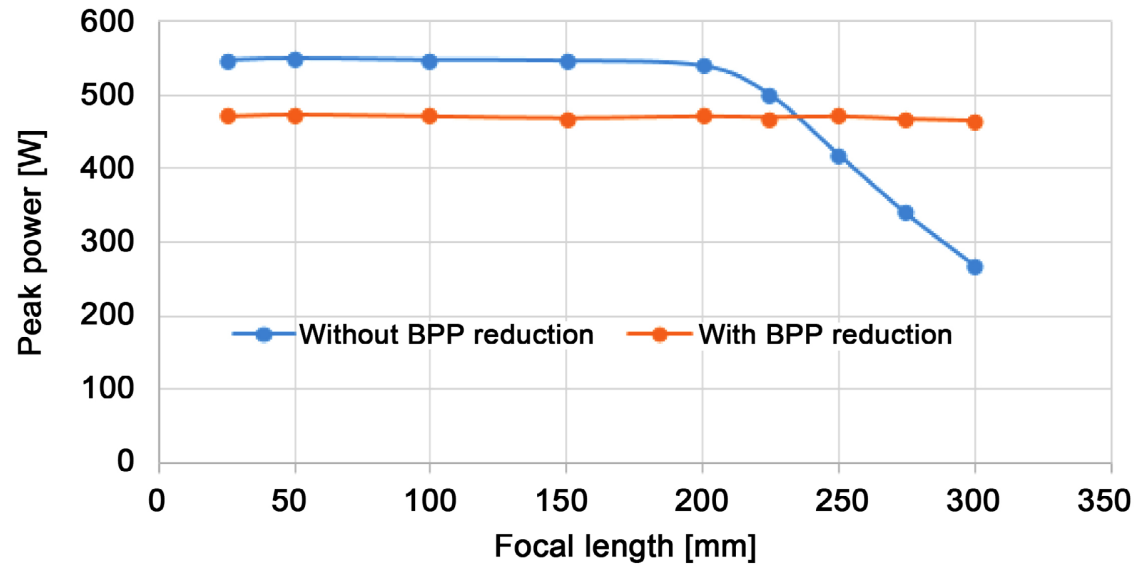

Figure 9. Measured power on the target versus focal length values with two different illuminator designs. 


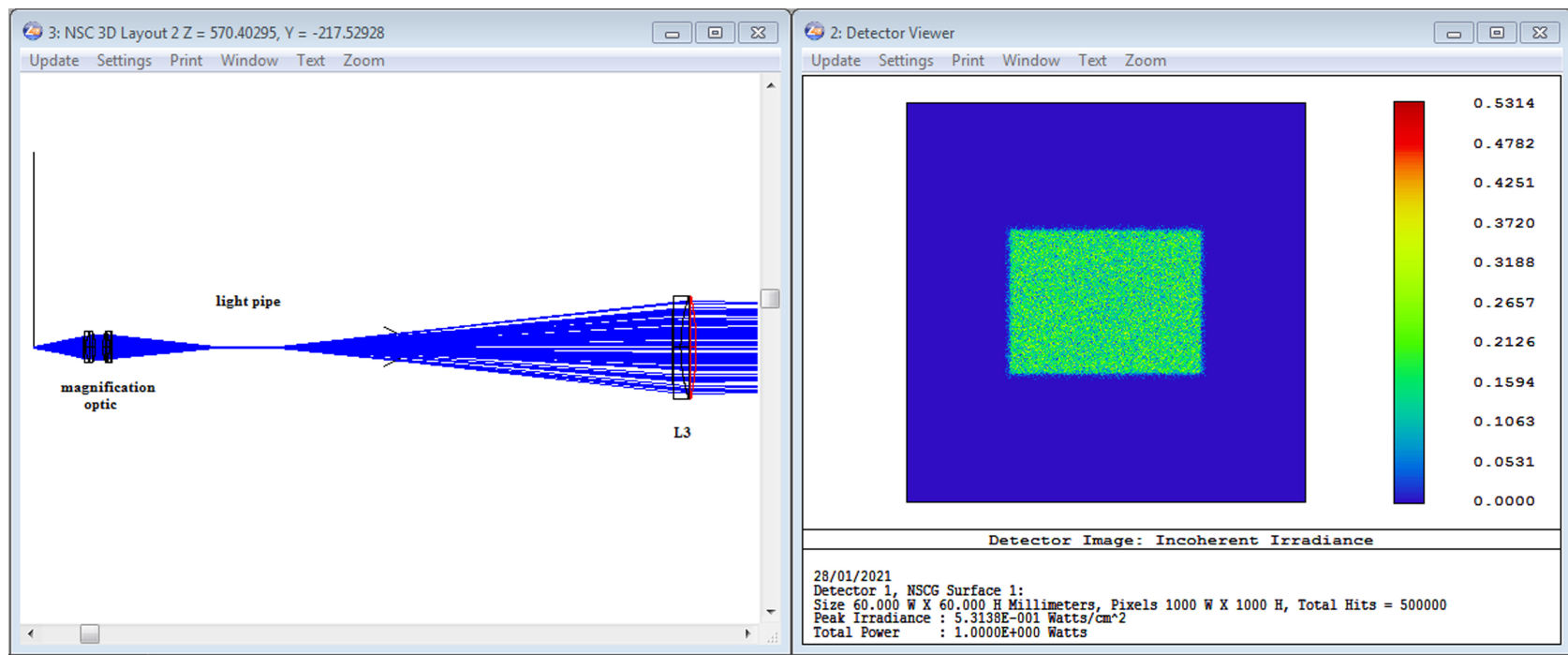

Figure 10. Beam propagation after fiber output of the laser diode based long range illuminator with reduced BPP.

BPP reduction setup described earlier (not shown on Figure 10). The beam forming light pipe remains unchanged as well as the zoom lens L3. In order to reduce the divergence angle after the light pipe input, a beam expanding optic with a magnification of 1.5 is placed between the fiber output and the pipe input. Negligible losses are measured when using AR coated lenses. With this operation, the beam diameter at the rod input is enlarged whereas the divergence is reduced by the same factor. It should be noticed that the beam forming light pipe works with total internal reflection corresponding to an invariant in the beam divergence between input and output.

The power on the target versus focus length is also measured for this new setup and reported on Figure 9. In this case we start from a lower value due to the losses of the beam shaping operations before fiber injection, however, no significant decrease is observed unlike the previous design and this all over the zoom focal range. The output power at maximum focal length of $300 \mathrm{~mm}$ could be increased from $270 \mathrm{~W}$ to $470 \mathrm{~W}$ thanks to the $\mathrm{BPP}$ reduction of the beam.

\section{Summary}

In this work, beam shaping operations are described allowing the reduction of the BPP of an SWIR laser diode stack in the kilowatt power range. Three operations are conducted on the beam such as spatial beam multiplexing consisting of free areas filling on the fast axis with a good efficiency of $93.1 \%$. The second operation is dedicated to polarization coupling in the slow axis direction of two sub beams with an efficiency of $95 \%$. Fiber injection is realized with this reduced BPP beam in a $600 \mu \mathrm{m}$ core fiber with NA $=0.22$ and a global efficiency of $83 \%$ without AR coatings. At the fiber output a magnification optic reduces the divergence before injection in a rectangular light pipe allowing high quality illumination beam. The zoom optic allows a tunable illumination divergence of $0.29^{\circ} \times 0.38^{\circ}$ to $3.4^{\circ} \times 4.5^{\circ}$. Thanks to the BPP reductions and even taking into 
account losses due to the additional components, the output power of the illuminator for the shortest divergences could be increased by $75 \%$ in comparison to the same illuminator without BPP reduction.

\section{Conflicts of Interest}

The author declares no conflicts of interest regarding the publication of this paper.

\section{References}

[1] Moor, N., Goings, J., Thiagarajan, P. and Caliva, B. (2017) Direct-Diode Laser Illumination and Pointing at Eye-Safe SWIR Wavelengths. Proceedings of SPIE, Vol. 10192, Laser Technology for Defense and Security XIII, Anaheim, California, 9-13 April 2017. https://doi.org/10.1117/12.2262721

[2] Dvinelis, E., Žukauskas, T., Kaušylas, M., Vizbaras, A., Vizbaras, K. and Vizbaras, D. (2016) Laser Illumination and EO Systems for Covert Surveillance from NIR to SWIR and beyond. Proceedings of SPIE, Vol. 9987, SPIE Security + Defense, Edinburgh, 26-29 September 2016. https://doi.org/10.1117/12.2238791

[3] Dayton, D., Gonglewski, J. and St-Arnauld, C. (2008) High Power Laser Diodes as LADAR Illuminators: Atmospheric Considerations. Proceedings of SPIE, Volume 7108, SPIE Remote Sensing, Cardiff, Wales, 15-18 September 2008. https://doi.org/10.1117/12.802549

[4] Basu, C., Wollweber-Meinhardt, M. and Roth, B. (2013) Lighting with Laser Diodes. Advanced Optical Technologies, 2, 213-321. https://doi.org/10.1515/aot-2013-0031

[5] Strohmaier, S., Tillkorn, C., Olschowsky, P. and Hostetler, J. (2010) High-Power, High-Brightness Direct-Diode Lasers. Optics and Photonics News, 21, 24-29. https://doi.org/10.1364/OPN.21.10.000024

[6] Li, Z., Li, T., Lu, P., Wang, Y., Zou, Y., Qu, Y., Bo, B., Liu, G. and Ma, X. (2012) A Survey of the High Power High Brightness Fiber Coupled Laser Diode. International Conference on Optoelectronics and Microelectronics, Changchun, 23-25 August 2012, 52-55. https://doi.org/10.1109/ICoOM.2012.6316214

[7] Köhler, B., Kissel, H., Flament, M., Wolf, P. and Biesenbach, J. (2010) High-Power Diode Laser Modules from $410 \mathrm{~nm}$ to $2200 \mathrm{~nm}$. Proceedings of SPIE, Vol. 7583, SPIE LASE, San Francisco, California, 23-28 January 2010. https://doi.org/10.1117/12.841846

[8] Lutz, Y., Bacher, E. and Schertzer, S. (2017) Accumulation Mode Laser Range-Gated Viewing in the Eye-Safe Spectral Region. Optics \& Laser Technology, 96, 1-6. https://doi.org/10.1016/j.optlastec.2017.04.031

[9] Ghasemi, S.H., Hantehzadeh, M.R., Sabbaghzadeh, J., Dorranian, D., Lafooti, M., Vatani, V., Rezaei-Nasirabad, R., Hemmati, A., Amidian, A.A. and Alavian, S.A. (2011) Beam Shaping Design for Coupling High Power Diode Laser Stack to Fiber. Applied Optics, 50, 2927-2930. https://doi.org/10.1364/AO.50.002927

[10] Lutz, Y. and Laurenzis, M. (2014) Beam Shaping of Laser Diode Stacks for Compact and Efficient Illumination Devices at the French-German Research Institute of Saint-Louis. Advanced Optical Technologies, 3, 179-185. https://doi.org/10.1515/aot-2013-0060

[11] Wu, Y., Dong, Z., Chen, Y., Qi, Y., Yuan, X., Qi, Y., Xu, L., Lin, X., Zou, Y. and Zhao, P. (2016) Beam Shaping for Kilowatt Fiber-Coupled Diode Lasers by Using One-Step Beam Cutting-Rotating of Prisms. Applied Optics, 55, 9769-9773. https://doi.org/10.1364/AO.55.009769 